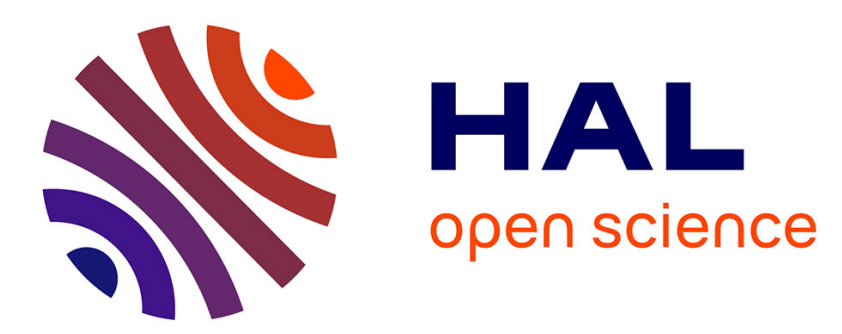

\title{
Bilayer bending elasticity measured by Fourier analysis of thermally excited surface undulations of flaccid vesicles
}

\author{
H. Engelhardt, H.P. Duwe, E. Sackmann
}

\section{- To cite this version:}

H. Engelhardt, H.P. Duwe, E. Sackmann. Bilayer bending elasticity measured by Fourier analysis of thermally excited surface undulations of flaccid vesicles. Journal de Physique Lettres, 1985, 46 (8), pp.395-400. 10.1051/jphyslet:01985004608039500 . jpa-00232534

HAL Id: jpa-00232534

https://hal.science/jpa-00232534

Submitted on 1 Jan 1985

HAL is a multi-disciplinary open access archive for the deposit and dissemination of scientific research documents, whether they are published or not. The documents may come from teaching and research institutions in France or abroad, or from public or private research centers.
L'archive ouverte pluridisciplinaire HAL, est destinée au dépôt et à la diffusion de documents scientifiques de niveau recherche, publiés ou non, émanant des établissements d'enseignement et de recherche français ou étrangers, des laboratoires publics ou privés. 
Classification

Physics Abstracts

$87.45-87.20$

\title{
Bilayer bending elasticity measured by Fourier analysis of thermally excited surface undulations of flaccid vesicles
}

\author{
H. Engelhardt, H. P. Duwe and E. Sackmann \\ Physics Department, Biophysics Group, Technical University Munich, D-8046 Garching, F.R.G.
}

(Reçu le 25 janvier 1985, révisé le 25 février, accepté le 25 février 1985)

\begin{abstract}
Résumé. - Nous mesurons l'élasticité de flexion de vésicules flasques par l'analyse de Fourier des ondulations thermiques de surface, jusqu'au $8^{\mathrm{e}}$ ordre. Les résultats sont expliqués par la théorie récente de Schneider et al. La constante élastique de flexion pour une bicouche fluide de lécithine $\left(K_{\mathrm{c}}=4 \times 10^{-13} \mathrm{erg}\right)$ est en bon accord avec celle des membranes d'érythrocytes, mais est presque d'un ordre de grandeur plus faible que celle donnée pour les mêmes lipides dans la littérature. Ce désaccord est attribué au fait que la valeur de $K_{\mathrm{c}}$ obtenue en ne considérant que le mode fondamental de déformation est systématiquement trop élevée.
\end{abstract}

\begin{abstract}
We measure the bending elasticity of flaccid vesicles by Fourier analysis of the thermally excited surface undulations up to the 8th order. The results are explained in terms of the recent theory of Schneider et al. The value of the bending elastic constant for fluid lecithin bilayers $\left(K_{\mathrm{c}}=4 \times\right.$ $10^{-13} \mathrm{erg}$ ) agrees well with that of erythrocyte membranes but is nearly an order of magnitude smaller than that reported for the same lipid in the literature. This discrepancy is attributed to the fact that the $K_{\mathrm{c}}$ value obtained by considering the lowest order mode of deformation alone is systematically too high.
\end{abstract}

The liquid crystalline nature of lipid bilayers and biomembranes is most clearly manifested in their curvature elasticity. There is growing evidence that it determines the shape of cells $[1,2]$ and plays also an essential role for the lateral organization of proteins in the membrane plane [3]. Measurements of the curvature elasticity of red cell membranes can give (1) insight into the coupling of the lipid protein bilayer to the cytoskeleton or (2) about the physiological state of erythrocyte membrane or (3) about the action of drugs on the cell deformability [8].

The bending elastic constant of pure lecithin bilayers has been measured by the micropipette technique [4], by evaluation of the mean square amplitude of the bending of cylindrical vesicles [5] and more recently by measurement of the time correlation function of the lowest order mode of the shape fluctuations of flaccid vesicles [6]. All these measurements lead to a value of the bending elastic constant of $K_{\mathrm{c}}=1-2 \times 10^{-12} \mathrm{erg}$ this value is at variance with the value obtained from measurements of the power spectrum of the erythrocyte flickering which leads to a lower value of $K_{\mathrm{c}}=2-7 \times 10^{-13} \mathrm{erg}[8,9]$.

In the present Letter we report measurements of the curvature elastic constant by time-dependent Fourier analysis of the shape fluctuations of flaccid vesicles using two-dimensional image analysis. The mean square amplitudes are measured as a function of the wave vectors up to the 8 th order. 
We obtain a curvature elastic constant of $K_{\mathrm{c}}=4 \times 10^{-13} \mathrm{erg}$ for fluid lecithin bilayers which agrees well with that reported for the red cell membrane $[7,8]$ but is at variance with the value of $K_{\mathrm{c}}$ reported for the lecithin vesicles $[1,4,6]$. We conclude that this discrepancy is due to the fact that the amplitude of the lowest order mode of the shape changes is modified by the gravitational force by second order effects or by a contribution of rotation of the vesicles becoming important at long relaxation times.

Giant vesicles are prepared between microslide and cover glass by swelling the lipid in excess water. The lipids dimyristoyl- (DMPC) and dipalmitoyl-phosphatidylcholine (DPPC) and the cholesterol are commercial products (Fluka). Water evaporation is prevented by sealing the gap between the glass plates with silicon grease. The width of the gap $(50-90 \mu \mathrm{m})$ is large compared to the diameter of the vesicle.

The experiments are performed with an inverted Zeiss Axiomat phase contrast microscope. For the data acquisition and data analysis a home-made image processing system is used. In an image storing device, each picture is represented by a $512 \times 512$ pixel block of 8 bit resolution obtained by digitizing normal TV-signal (BAS) with a rate of $10 \mathrm{MHz}$. The image storing hardware is controlled by a microcomputer using a MC $68000 \mathrm{CPU}$.

The present method is based on the Fourier analysis of the contour $R(\varphi)$ of the vesicles as observed in the image plane (cf. Fig. 1). $R(\varphi)$ is the momentaneous radius of the contour as measured from the centre of the average contour $R_{0}(\varphi)$ and $\varphi$ is the rotational angle in the image plane.

Images of the vesicles are taken at a rate of 25 frames s$^{-1}$ (European norm) and stored on a U-Matic videorecorder (Sony). For the analysis of the shape changes, images taken at time


Fig. 1. - Photomicrograph of vesicle of $94 \%$ DMPC and $6 \%$ cholesterol taken at different times : $t$ and $t+5$ seconds, respectively. The contour $R(\varphi)$ is marked by the white line drawn by the cursor. The traces at the bottom show the transmitted light intensity along the cross sections of the vesicles. 
intervals of $0.5 \mathrm{~s}$ are transferred from the recorder to the image processing system. The second step consists in the search and storage of the coordinates of the contours $R(\varphi)$ of the vesicles which is defined by the minimum of the intensity (cf. Fig. 1). For that purpose a cursor is put at a certain point $R(\varphi)$ and is then proceeding on the minimum. The fluctuations of the contour $\Delta R(\varphi)=$ $R(\varphi)-R_{0}(\varphi)$ is then expanded in a Fourier series as

$$
\Delta R(\varphi)=\sum_{q} U_{q} \mathrm{e}^{-i q \varphi} ; \quad U_{q}=\frac{1}{2 \pi} \int_{0}^{2 \pi} R(\varphi) \mathrm{e}^{i q \varphi} \mathrm{d} \varphi .
$$

The mean square values of the amplitudes, $\left\langle U_{q}^{2}\right\rangle$, are determined by averaging over a selected number, $N$, of pictures (typically $N=39$ ) corresponding to an observation time of $N \times 0.5$ seconds.

A typical result is shown in figure 2. The top shows the deviation $\Delta R(\varphi)$ of the contour from the average of $R_{0}(\varphi)$. At the lower left corner the square root of $\left\langle U_{q}^{2}\right\rangle$ is plotted as a function of $q$. The determination of the bending elastic constants from the mean square amplitudes $\left\langle U_{q}^{2}\right\rangle$ is discussed below.

The bending elastic constant is obtained from the mean square amplitudes of $\left\langle U_{q}^{2}\right\rangle$ defined in equation (1) following the theory of Schneider et al. [6]. These authors extended previous theories


Fig. 2. - Top : Deviation $\Delta R(\varphi)$ of contour from average radius $R_{0}(\varphi)$ for a vesicle of DMPC with 6 mole $\%$ of cholesterol. Temperature $30^{\circ} \mathrm{C}$ average radius $a=9.2 \mu \mathrm{m}$. Bottom left : Mean value of Fourier amplitude $\left\langle U_{q}\right\rangle$ plotted as a function of the wave vector $q$. The dotted horizontal line defines the noise level. The amplitudes $\left\langle U_{l}\right\rangle$ have been discarded since they contain translations of the whole vesicle. Bottom right : Curvature elastic constant, $K_{\mathrm{c}}$ plotted as a function of $q$. Note that $K_{\mathrm{c}}$ is much larger for $q=2$ than for $3 \leqslant q \leqslant 8$. 
$[7,8]$ on the thermal excited surface undulations of infinitely large planar bilayers to the situation of closed flaccid vesicles. These can assume a manifold of states at constant area and volume which are expected to be attained by thermal excitation at room temperature if the surface tension or the shear elasticity of the bilayers as well as gravitational forces can be neglected. Under these conditions the change in the vesicle shape is completely described in terms of displacements $U(\theta, \varphi)$ in the normal direction which may be expanded in spherical harmonics

$$
U(\theta, \varphi)=\sum_{l m} U_{l m} Y_{l m}(\theta, \varphi) ; \quad Y_{l m}(\theta, \varphi)=\sqrt{\frac{2 l+1}{4 \pi} \frac{(l+|m|) !}{(l-|m|) !}} P_{l}^{m}(\cos \theta) \mathrm{e}^{i m \varphi}
$$

where $\theta$ and $\varphi$ define the altitude and latitude of a point on the sphere, respectively, and where $U_{l m}(t)$ are the time-dependent amplitudes. According to Schneider et al. the excess curvature elastic energy of the fluctuating vesicle is given to second order by

$$
F=\sum_{l m} \frac{K_{\mathrm{c}}}{2}(l-1)^{2}(l+2)^{2} \frac{U_{l m}^{2}}{a^{2}}
$$

where $a$ is the average radius of the vesicle. $a$ is obtained by averaging over all values $R_{0}(\varphi)$ and corresponds to the zeroth order of $R_{0}(\varphi)$.

Equation (2) is based on a linearized elastic theory which does not imply tangential deformations. This approximation is certainly valid for wavelengths large compared to molecular dimensions and tangential effects may therefore be neglected in our case.

All $2 l+1$ modes belonging to the same quantum number $l$ are degenerated. According to the equipartition theorem the mean square amplitudes are then given by

$$
\left\langle U_{l m}^{2}\right\rangle=\frac{k_{\mathrm{B}} T a^{2}}{(l-1)^{2}(l+2)^{2} K_{\mathrm{c}}} .
$$

Since the mean square amplitudes $\left\langle U_{q}^{2}\right\rangle$ of equation (1) may be expressed in terms of the corresponding values, $\left\langle U_{l m}^{2}\right\rangle$ of the spherical harmonics expansion it is possible to obtain the elastic constant $K_{\mathrm{c}}$ from the Fourier analysis of the contour $R(\varphi)$ alone. Consider a section through the centre of the vesicle which is defined by the fixed angle $\theta=\pi / 2$. The displacement $U(\theta, \varphi)$ of the corresponding cross section may be expanded according to equation (1). For $\theta=\pi / 2$ it follows :

$$
U_{q}=\frac{1}{2 \pi} \int_{0}^{2 \pi} \sum_{l m} U_{l m} Y_{l m}(\pi / 2, \varphi) \mathrm{e}^{i q \varphi} \mathrm{d} \varphi
$$

For a different section through the centre of the vesicle, $U_{q}$ is obtained in the usual way [10] by rotation of the coordinate system by the Eulerian angles $\alpha, \beta, \gamma$ according to

$$
U_{q}=\frac{1}{2 \pi} \int_{0}^{2 \pi} \sum_{l m} U_{l m} \sum_{k} D_{k m}^{l}(\alpha \beta \gamma) Y_{l k}\left(\pi / 2, \varphi^{\prime}\right) \mathrm{e}^{i q \varphi^{\prime}} \mathrm{d} \varphi^{\prime}
$$

where $D_{k m}^{l}(\alpha \beta \gamma)$ is the Wigner rotation matrix. The mean square amplitude $\left\langle U_{q}^{2}\right\rangle$ is obtained by integration over $\varphi^{\prime}$ and by averaging over all angles $\alpha, \beta, \gamma$. Together with equation (3) one obtains finally

$$
\left\langle U_{q}^{2}\right\rangle=\frac{k_{\mathrm{B}} T a^{2}}{K_{\mathrm{c}}} \sum_{l \geqslant q}\left(\frac{N_{l q} P_{l}^{q}(0)}{(l+2)(l-1)}\right)^{2}
$$


where $N_{l q}$ are normalization constants. The sum on the right side is rapidly converging and has been calculated numerically up to the 34 th order. With equation $(6) K_{\mathrm{c}}$ can now be determined for each measured mean square amplitude $\left\langle U_{q}^{2}\right\rangle$ since $a^{2}$ can also be measured.

The bottom trace of figure 2 shows the result of this evaluation procedure for the cholesterol containing DMPC vesicle. The plot of the reciprocal elastic constant $K_{\mathrm{c}}$ as a function of $q$ increases by about a factor of 5 from $q=2$ to $q=3$, reaches a constant plateau at $3 \leqslant q \leqslant 8$ and decreases again at $q \geqslant 9$. Such a behaviour has been observed for most vesicles studied although the limits of the plateau may be shifted.

The decrease at $q \geqslant 9$ can be attributed to the rise-time, $\tau_{\mathrm{r}}$, of the TV-tube ( $\tau_{\mathrm{r}} \approx 0.05 \mathrm{~s}$ ). According to Schneider et al. [6] the response time, $\tau_{l m}$, of the surface undulation decreases rapidly with increasing $l$ and becomes $\approx 0.075 \mathrm{~s}$ for $l=8$. The good agreement of the $K_{\mathrm{c}}$ values at $3 \leqslant q \leqslant 8$ provides very strong evidence that the amplitudes of the higher order modes yield the true value of the elastic constant while that obtained from the 2 nd order mode is systematically too high. In fact the elastic constant obtained for $q=2\left(K_{\mathrm{c}}=8 \times 10^{-13} \mathrm{erg}\right)$ is comparable to that obtained in references [4-6].

There are several factors leading to too high $K_{\mathrm{c}}$ values by evaluation of the low order modes. The gravitational force exerts two effects. Firstly, it restricts the long wavelength displacements. Thus, the thermal energy would be just sufficient to excite amplitudes of $1 \mu \mathrm{m}$ against the gravitational force (bilayer density of $1.025 \mathrm{~g} / \mathrm{cm}^{3}$ ). In addition gravitational force will cause a coupling of the rotation and the deformation of the low order modes due to the intrinsic high amplitudes of the latter. Another factor leading to too high values for small $q$ 's is the suppression of the surface undulations owing to non-linear surface tension effects [11]. Finally it should be noted that the sampling time of $0.5 \mathrm{~s}$ is comparable to the correlation time $\tau_{l m}$ for small $q$ 's. However this effect can be neglected because the $K_{\mathrm{c}}$ values did not change if a longer sampling time was used.

The method has been applied to several types of bilayers. The results for vesicles of pure DMPC, of a solution of 6 mole \% of cholesterol in DMPC and of a 1:1 mixture of DMPC and DPPC are given in table I. For each system studied, the bending stiffness of several different vesicles which appeared as very thin-walled were measured. The $K_{c}$ values for the mixed system in particular those for the cholesterol containing DMPC-vesicles vary only by about $30 \%$ for the different vesicles. This shows that the Fourier analysis of the contours of fluctuating vesicles enables rather accurate measurements of the bending elastic constant of giant vesicles. The method can be considerably improved if the dynamic range of the method is increased so that the amplitudes can be measured to higher orders $(q>8)$. It appears that all disturbing influences such as gravitational forces, non-linear effects or rotational motions suppress the amplitudes of the undulations and exert a stronger effect on the long wavelength modes. We thus conclude that the $K_{\mathrm{c}}$ values obtained by analysing the higher order modes $(q \geqslant 3)$ correspond to the true bending elastic constants of the bilayer while the lowest order fluctuation mode yields systematically too high values of $K_{\mathrm{c}}$.

Table I. - Bending elastic constants of thin-walled vesicles.

\begin{tabular}{|l|c|c|c|}
\hline \multicolumn{1}{|c|}{ System } & Number of vesicles & $T\left({ }^{\circ} \mathrm{C}\right)$ & $K_{\mathrm{c}}\left(10^{-13} \mathrm{erg}\right)$ \\
\hline DMPC & 4 & 26 & $3.5-6.5$ \\
\hline DMPC $+6 \%$ cholesterol & 6 & 29 & $2.8-3.7$ \\
\hline DMPC/DPPC & 2 & 30 & $3.8-4.9$ \\
\hline
\end{tabular}


The close agreement of $K_{\mathrm{c}}$ of different vesicles is surprising since it is expected to be proportional to the number of coupled bilayers forming the shell. It thus appears that the vesicles of DMPC/ cholesterol and DMPC/DPPC studied were all single-walled or were at least formed by the same number of bilayers. For pure DMPC $K_{\mathrm{c}}$ varies by about a factor of two for the different vesicles (cf. Table I) which could be due to differences in the shell thickness. It should be noted, however, that the pure DMPC does not form such large vesicles as the mixtures and the values of $K_{\mathrm{c}}$ obtained are less accurate. On the other side vesicles which appeared really thick-walled by visual inspection exhibit indeed very small amplitudes corresponding to a bending stiffness of $K_{\mathrm{c}} \cong 100 \times 10^{-13} \mathrm{erg}$.

\section{Acknowledgments.}

The work was supported by the Deutsche Forschungsgemeinschaft (Sa 246/15-1) and by the Fond der Chemie.

\section{References}

[1] Deuling, H. J. and Helfrich, W., Biophys. J. 13 (1980) 941.

[2] Svetina, S. and Zeks, B., Biomed. Biochim. Acta 42 (1983) 86.

[3] Sackmann, E., Kotulla and Heiszler, F. J., Can. J. Biochem. Cell Biol. 62 (1984) 778.

[4] Evans, E. A., Biophys. J. 43 (1983) 27.

[5] Servus, R. M., Harbich, W. and Helfrich, W., Biochem. Biophys. Acta 436 (1976) 900.

[6] Schneider, M. B., Jenkins, J. T. and WebB, W. W., J. Physique 45 (1984) 1457.

[7] Kramer, L., J. Chem. Phys. 55 (1971) 2097.

[8] Brochard, F. and Lennon, J. F., J. Physique 36 (1975) 1035.

[9] Fricke, K. and Sackmann, E., Biochim. Biophys. Acta.

[10] Davydow, A. S., Quantenmechanik (Berlin Dt. Verlag d. Wiss.) 1978.

[11] Brochard, F., De Gennes, P. G. and Pfeuty, P., J. Physique 37 (1976) 1099. 